\title{
Extreme thermopower anisotropy and interchain transport in the quasi-one-dimensional metal $\mathrm{Li}_{0.9} \mathrm{Mo}_{6} \mathrm{O}_{17}$
}

\author{
J. L. Cohn, ${ }^{1, *}$ S. Moshfeghyeganeh,${ }^{1}$ C. A. M. dos Santos, ${ }^{2}$ and J. J. Neumeier ${ }^{3}$ \\ ${ }^{1}$ Department of Physics, University of Miami, Coral Gables, FL 33124 \\ ${ }^{2}$ Escola de Engenharia de Lorena - USP, P. O. Box 116, Lorena-SP, 12602-810, Brazil \\ ${ }^{3}$ Department of Physics, Montana State University, Bozeman, Montana 59717
}

\begin{abstract}
Thermopower and electrical resistivity measurements transverse to the conducting chains of the quasi-one-dimensional metal $\mathrm{Li}_{0.9} \mathrm{Mo}_{6} \mathrm{O}_{17}$ are reported in the temperature range $5 \mathrm{~K} \leq T \leq 500 \mathrm{~K}$. For $T \geq 400 \mathrm{~K}$ the interchain transport is determined by thermal excitation of charge carriers from a valence band $\sim 0.14 \mathrm{eV}$ below the Fermi level, giving rise to a large, $p$-type thermopower that coincides with a small, $n$-type thermopower along the chains. This dichotomy - semiconductorlike in one direction and metallic in a mutually perpendicular direction - gives rise to substantial transverse thermoelectric (TE) effects and a transverse TE figure of merit among the largest known for a single compound.
\end{abstract}

Conducting materials with highly anisotropic Seebeck coefficients (thermoelectric powers or TEPs) are potentially useful in transverse thermoelectric applications for energy detection and cooling [1-3]. Bulk conductors for which the TEPs in different crystallographic directions have opposite signs and yield a large magnitude for their difference $(\Delta S \geq 200 \mu \mathrm{V} / \mathrm{K})$ are quite rare $[3,4]$, thus recent developments have focused on artificial synthesis of stacked bulk materials $[1,2]$ or semiconductor heterostructures [3] to achieve large Seebeck anisotropy. Here we present transport measurements on the quasi-one-dimensional (q1D) metal, $\mathrm{Li}_{0.9} \mathrm{Mo}_{6} \mathrm{O}_{17}$ known as "lithium purple bronze" (LiPB), that reveal a surprisingly simple mechanism for extreme Seebeck anisotropy in a bulk conductor. Direct electron transfer between the q1D metallic chains of this material is sufficiently weak that interchain transport above $400 \mathrm{~K}$ is predominated by thermal activation of valence band states $\left(\sim 0.14 \mathrm{eV}\right.$ below $\left.E_{F}\right)$, yielding a large, $p$-type interchain Seebeck coefficient that coexists with $n$-type metallic behavior confined along the q1D chains. A substantial transverse Peltier effect is demonstrated. These ingredients may exist in other materials or might possibly be engineered to develop transverse thermoelectrics based on a single compound.

A resistivity that is metallic at low-temperature and decreases anomalously at high temperatures is a ubiquitous characteristic of transport transverse to the planes or chains of many q2D [5-8] and q1D metals [9-11], respectively. It is generally accepted that this behavior is due to the onset of an additional conduction mechanism in parallel with band transport, possibly related to interplane or -chain defects (e.g. resonant tunneling) [12].

Much less is known about the TEP transverse to the planes or chains of such materials, partly because TEP measurements are difficult to perform in small single crystals for which the transverse transport directions have very small dimensions (e.g., thin platelet or needlelike habits). In the few compounds where transverse
TEP measurements have been reported [13-18], high anisotropy has not been observed.

$\mathrm{Li}_{0.9} \mathrm{Mo}_{6} \mathrm{O}_{17}$ known as "lithium purple bronze" (LiPB), is a low-temperature superconductor $\left(T_{c} \approx 2 \mathrm{~K}\right)$ first synthesized and studied in the 1980s [19-21]. It has attracted interest more recently for its quasi-one dimensionality and Luttinger-liquid candidacy [22-28]. Crystal growth $[20,25]$ and transport properties along the chains (crystallographic $b$ axis) for crystals similar to those discussed here have been presented elsewhere [29, 30]. The resistivity anisotropy of LiPB is approximately [31] $\rho_{b}: \rho_{c}: \rho_{a}=1: 80: 1600$. Single-crystal specimens were oriented by x-ray diffraction and cut/polished into thin rectangular plates with the thinnest dimension (along the $a$ axis) typically $40-80 \mu \mathrm{m}$. The $b c$-plane dimensions were typically $0.4 \times 1.0 \mathrm{~mm}$ with the longest dimension coinciding with the transport axis ( $b$ or $c$ direction). Electrical contacts were made with $\mathrm{Au}$ leads attached with silver epoxy. Current contacts covered the specimen ends and voltage contacts encircled the crystals across both large faces and the sides. For thermopower measurements, specimens were suspended from a $\mathrm{Cu}$ heat sink with silver epoxy and affixed with a heater and $25-\mu \mathrm{m}$ diameter differential chromel-constantan thermocouple, both attached with stycast epoxy. Separate radiationshielded vacuum probes were employed for the cryogenic and high- $T$ (> $320 \mathrm{~K}$ ) measurements.

Figure 1 (a) shows for two crystals the interchain resistivity and TEP, $\rho_{c}(T)$ and $S_{c}(T)$, along with the intrachain TEP, $S_{b}(T)$, for two different crystals [29]. Additional $c$-axis data for two more crystals can be found in the Supplementary Material [32]. The increase in all three coefficients below $30 \mathrm{~K}$ has been discussed extensively elsewhere [29] and may be associated with localization, dimensional crossover or the development of unconventional (e.g., electronically-driven) charge densitywave order $[24,25]$. The focus of the present work is the interchain TEP in the region $T>40 \mathrm{~K}$ where it rises sharply with increasing $T$, coincident with a deviation 


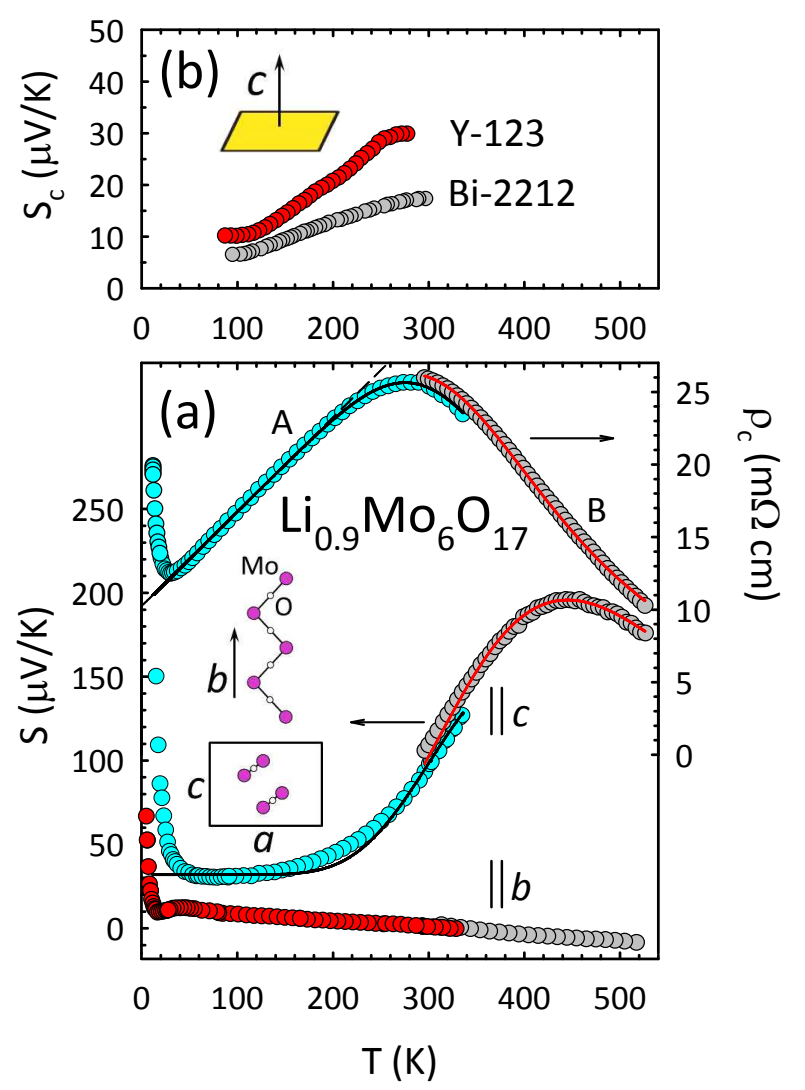

FIG. 1. (color online) (a) LiPB interchain (c-axis) thermopowers (left ordinate) and resistivities (right ordinate) for two crystals (labeled A and B), and intrachain ( $b$-axis) thermopowers for two different crystals. Solid curves through the $c$-axis data are fits to the parallel conduction model discussed in the text; parameters for the semiconducting component are listed in Table I. Dashed line is a linear-least-squares fit to the low- $T \rho_{c}(T)$ (see text). Inset: orientation of the crystallographic axes with respect to the q1D Mo-O chains (2 per unit cell). (b) interplane (c-axis) thermopowers for $\mathrm{YBa}_{2} \mathrm{Cu}_{3} \mathrm{O}_{7}$ (Y-123, Ref. 16) and $\mathrm{Bi}_{2} \mathrm{Sr}_{2} \mathrm{CaCu}_{2} \mathrm{O}_{8}$ (Bi-2212, Ref. 17).

of $\rho_{c}(T)$ from it's low- $T$, linear- $T$ behavior (dashed line, Fig. 1). The intrachain TEP is linear-in- $T$, modest in magnitude, and becomes negative above $300 \mathrm{~K}$, consistent with electron-like carrier diffusion as noted previously [29], and extended here to $520 \mathrm{~K}$.

Several features of $S_{c}(T)$ are noteworthy. It remains positive throughout the temperature range. In the linear$T$ regime of $\rho_{c}(40 \mathrm{~K}<T<140 \mathrm{~K}), S_{c}$ is nearly $T$ independent at $\sim 32 \mu \mathrm{V} / \mathrm{K}$ and essentially the same for all crystals measured. Near the maximum in $S_{c}$ at $T \simeq 440 \mathrm{~K}, \Delta S=S_{c}-S_{b} \geq 200 \mu \mathrm{V} / \mathrm{K}$. The interchain transport in LiPB is incoherent, the metallic character of $\rho_{c}$ in the lower- $T$ regime likely reflecting the intrachain scattering rate [33], consistent with very weak and indirect interchain hopping [28]. The nearly constant TEP at low- $T$ is a characteristic of narrow-band hopping [34]. As for the increase in $S_{c}$ to very large values at higher $T$,

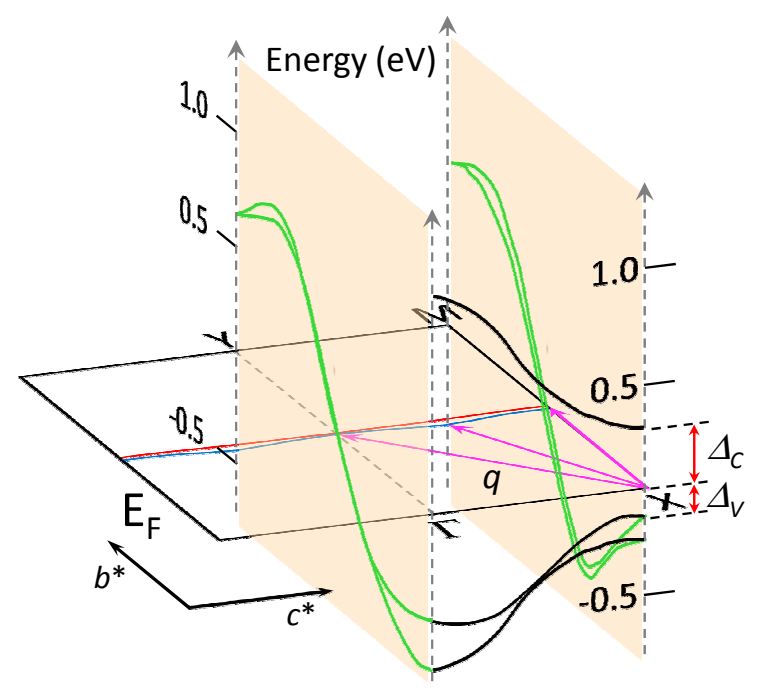

FIG. 2. (color online) Bands along main symmetry directions (green: along $b^{*}$, black: along $c^{*}$ ) and projected Fermi surface (red and blue curves) in the $b^{*} c^{*}$ plane for LiPB (adapted from Ref. 28). $\Delta_{V}$ and $\Delta_{C}$ are excitation energies for valenceand conduction-band states, respectively, dispersing along $c^{*}$ near the X-point of the Brillouin zone. Indirect transitions between these states and the Fermi surface are possible through thermal excitation and absorbtion of a phonon with sufficient momentum along $b^{*}$ (wavevectors for excitation of valenceband states are represented by pink arrows).

it is instructive to compare with the behavior found for the interplane (c-axis) TEPs of the q2D metals $[16,17]$, $\mathrm{YBa}_{2} \mathrm{Cu}_{3} \mathrm{O}_{7}$ and $\mathrm{Bi}_{2} \mathrm{Sr}_{2} \mathrm{CaCu}_{2} \mathrm{O}_{8}$ [Fig. 1 (b)]. The TEPs of the latter materials, truncated by the onset of superconductivity at low- $T$, also rise for $T \gtrsim 120 \mathrm{~K}$, but their overall increases $(\lesssim 20 \mu \mathrm{V} / \mathrm{K})$ are substantially smaller than for LiPB and a tendency toward saturation is evident at the highest $T$. Their upturns are plausibly attributed to the onset of interplane tunneling, though theoretical work [35] has not yet treated the TEP within a model that incorporates resonant tunneling through defects [12]. The upturn in the interchain TEP for LiPB is qualitatively and quantitatively different.

We propose that this difference has its origin in the LiPB band structure which is distinguished from these other compounds by the presence of valence and conduction bands in close energy proximity to $E_{F}$ and with sufficient dispersion for interchain momentum so as to become increasingly important for interchain transport with increasing $T$. Figure 2 shows the calculated band structure $[21,23,27,28]$ within the $b^{*}-c^{*}$ plane (dispersion along $a^{*}$ is negligible). The projected Fermi surface (blue and red curves) is also shown. Electrons in valence bands dispersing along $c^{*}$ (black curves) can make indirect, interband transitions to states at $E_{F}$ through thermal excitation $\left(\Delta_{V}\right.$, Fig. 2) and absorption of phonons with sufficient momenta $(q)$ along the $b^{*}$ direction (pink 
arrows, Fig. 2). Similarly, electrons at $E_{F}$ in states dispersing along $b^{*}$ can be thermally excited $\left(\Delta_{C}\right)$ to the lowest-lying interchain conduction band above $E_{F}$ near the X-point by absorbing phonons with opposite momenta. These phonons, with momenta $q \lesssim \sqrt{2} \pi / 2 b$ and energies $\hbar q v \lesssim 6 \mathrm{meV}$ (assuming a dispersionless, acoustic phonon with velocity $v=3 \mathrm{~km} / \mathrm{s}$ ), will be excited in large numbers at room temperature and above. The activation energies, from averaging the various band structure calculations, are $\Delta_{V}=0.16 \mathrm{eV}$ and $\Delta_{C}=0.20 \mathrm{eV}$. With $\Delta_{V}<\Delta_{C}$ a $p$-type thermopower should result.

These observations motivate an interpretation of the interchain transport in LiPB that reflects parallel conduction through band-like states $(l o)$, predominant at $T \lesssim 140 \mathrm{~K}$, and a thermally activated, semiconductor contribution $(h i)$, predominant at $T>300 \mathrm{~K}$,

$$
\begin{aligned}
& \sigma=\sigma^{l o}+\sigma^{h i} \\
& S=\left(\sigma^{l o} / \sigma\right) S^{l o}+\left(\sigma^{h i} / \sigma\right) S^{h i} .
\end{aligned}
$$

The low- $T$ TEP is taken as $S^{l o}=32 \mu \mathrm{V} / \mathrm{K}$, independent of $T$ as motivated by the $S_{c}(T)$ data, and $\sigma^{l o}=1 /(A+B T)$ from linear-least-squares fits (dashed lines, Fig. 1) to the $\rho_{c}(T)$ data in the range $40 \mathrm{~K} \leq$ $T \leq 140 \mathrm{~K}$. For the high- $T$ contribution we first tried a single semiconductor component to minimize the number of free parameters: $\sigma^{h i}=C \exp \left(-E_{\sigma} / k_{B} T\right)$ and $S^{h i}=\left(k_{B} /|e|\right)\left(E_{S} / k_{B} T+D\right)$, where $C$ and $D$ are constants and $E_{\sigma}$ and $E_{S}$ are activation energies. The solid curves through the $\rho_{c}(T)$ and $S_{c}(T)$ data (Fig. 1) demonstrate the agreement achieved with Eq. (1) throughout the range $T \geq 40 \mathrm{~K}$ using these simple forms for $\sigma^{h i}$ and $S^{h i}$ (parameter values are listed in Table I). The discrepancy between the computed and measured TEPs in the transition region (near $200 \mathrm{~K}$ ) may reflect our neglect of a tunneling contribution, like that observed for the cuprates [Fig. 1 (b)], but has negligible impact on the fitting at high- $T$ if, as in the latter materials, this contribution adds a small constant. The average activation energies found for the four crystals (with s.d. uncertainties) are: $E_{\sigma}=0.15 \pm 0.01 \mathrm{eV}$ and $E_{S}=0.24 \pm 0.01 \mathrm{eV}$.

The observation $E_{S}>E_{\sigma}$ is incompatible with singleband $\left(E_{S}=E_{\sigma}\right)$ and intrinsic two-band $\left(E_{S} \leq E_{\sigma}\right)$ semiconductor conduction for the high- $T$ component, but

TABLE I. Fitting parameters for the semiconductor, high- $T$ component $(h i)$ of the parallel conduction model discussed in the text.

\begin{tabular}{ccccc}
\hline \hline Specimen & $C\left(\Omega^{-1} \mathrm{~cm}^{-1}\right)$ & $E_{\sigma}(\mathrm{eV})$ & $D$ & $E_{S}(\mathrm{eV})$ \\
$\mathrm{A}$ & $2.40 \times 10^{3}$ & 0.155 & -3.00 & 0.220 \\
$\mathrm{~B}$ & $2.41 \times 10^{3}$ & 0.159 & -2.90 & 0.250 \\
$\mathrm{C}$ & $1.60 \times 10^{3}$ & 0.146 & -2.68 & 0.241 \\
$\mathrm{D}$ & $1.85 \times 10^{3}$ & 0.146 & -2.15 & 0.241 \\
\hline \hline
\end{tabular}

is naturally explained [32], consistent with the band structure, if both valence and conduction band states are excited with differing activation energies, $\Delta_{V}$ and $\Delta_{C}$. Analyzing the data with a three-component model, low- $T$ metallic and two high- $T$ semiconducting contributions [32], yields average energies $\Delta_{V} \simeq 0.14 \mathrm{eV}$ and $\Delta_{C} \simeq 0.23 \mathrm{eV}$, i.e. nearly the same as $E_{\sigma}$ and $E_{S}$ in the two-component model. The interband transitions evidently serve as the predominant mechanism for interchain (c-axis) transport above $400 \mathrm{~K}$ where the semiconducting components represent more than $50 \%$ of the total conductivity [32]. In this regime LiPB thus behaves simultaneously as a $p$-type semiconductor and $n$-type metal along mutually perpendicular directions, leading to its high Seebeck anisotropy.

To test for the transverse Peltier effect, a rectangular specimen $\left(x \times y \times z=1.9 \times 0.9 \times 0.2 \mathrm{~mm}^{3}\right)$ with its long axis at an angle $\alpha=32^{\circ}$ to the $b$ axis (Fig. 3, inset), was mounted with one edge thermally anchored to a copper heat sink in vacuum; current was applied along the $x$ direction. Cooling or warming at the free edge of the crystal, monitored by a differential thermocouple with junctions along the $y$ direction (separation $d \simeq 0.4 \mathrm{~mm}$ ), was induced by forward or reverse current, respectively, and was linear in the current (Fig. 3). The averaged temperature gradient, $d T / d y=[\Delta T(I+)-\Delta T(I-)] / 2 d$, is shown as a function of the average specimen temperature in Fig. 4 for fixed values of the current. The dashed curves in Fig. 4 were computed using $\Delta S$ [interpolated

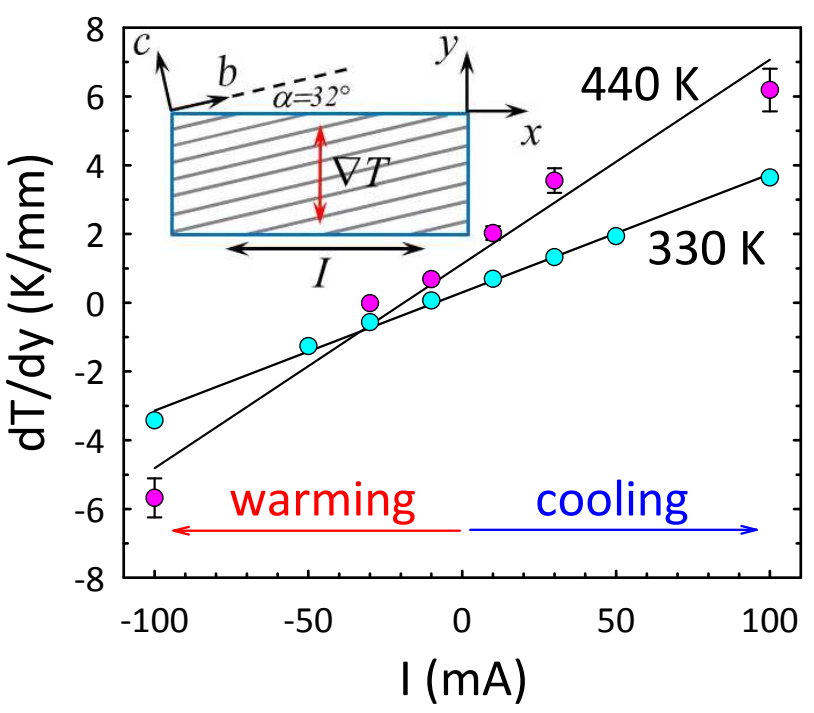

FIG. 3. (color online) Current dependence of the transverse Peltier-induced temperature gradient for a LiPB crystal at $T=330 \mathrm{~K}$ and $440 \mathrm{~K}$. Error bars reflect uncertainty due to slow oscillations in the $\mathrm{Cu}$ block temperature $(\leq 0.1 \mathrm{~K})$, particularly at the highest $T$. The inset shows the orientation of current and heat flow relative to the specimen $(x-y)$ and crystallographic $(b-c)$ axes. 


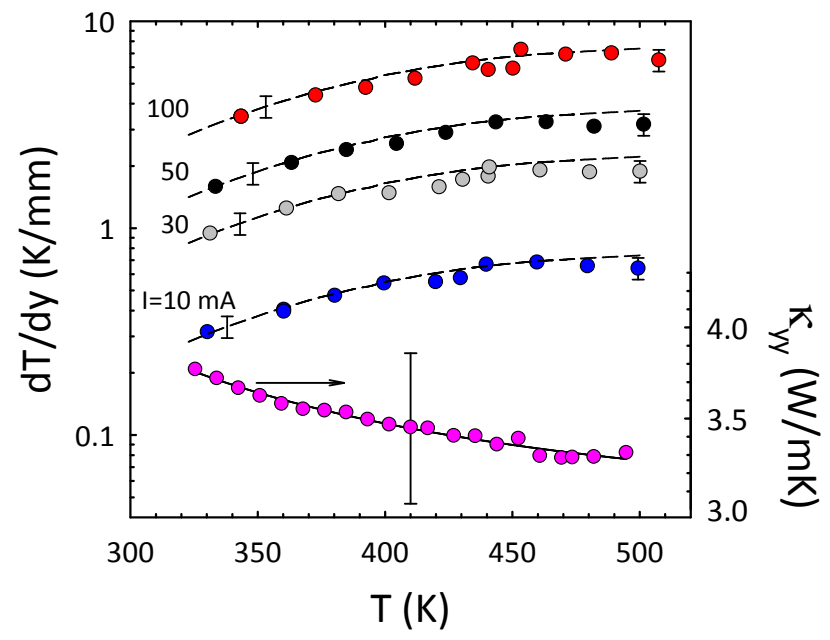

FIG. 4. (color online) $d T / d y$ (averaged for,+- current) $v$ s. the average specimen temperature at fixed applied currents (left ordinate), and $\kappa_{y y}(T)$ (right ordinate). The dashed curves are computed (see text) using $\kappa_{y y}$ and $\Delta S$ interpolated from Fig. 1 (a). Error bars for the data and computed curves $(12 \%)$ are determined by uncertainty in the thermocouple junction separation. The $\kappa_{y y}$ data were corrected for radiation losses and conduction through the leads estimated from direct measurements on similar specimens suspended from their leads. These corrections amounted to $6 \%$ at $300 \mathrm{~K}$ and $30 \%$ at $500 \mathrm{~K}$.

from data for crystal B, Fig. 1 (a)], the transverse thermal conductivity $\left(\kappa_{y y}\right)$ measured in a separate experiment with a heater attached to the free end of the specimen (Fig. 4, right ordinate), and the heat flux equation [2], $d T / d y=\left(T S_{x y} / \kappa_{y y}\right) j$, where $S_{x y}=(1 / 2) \Delta S \sin (2 \alpha)$ and $j$ is the current density. The latter expression ignores a Joule heating term (varying as $j^{2}$ ), justified by the linearity of $d T / d y$ with current noted above. Because LiPB has a rather low thermal conductivity along the $c$ axis [30], the transverse thermoelectric figure of merit is among the largest known for a single-phase material [36], $Z_{x y} T=T S_{x y}^{2} /\left(\rho_{x x} \kappa_{y y}\right) \simeq 0.024 \pm 0.007$ at $450 \mathrm{~K}$ [37].

LiPB may itself prove useful in converting waste heat to electrical power or in energy detection. Given that the features underlying its extreme Seebeck anisotropy appear fairly generic - low-dimensional, metallic electronic structure and dispersing bands for the transverse direction in close proximity to $E_{F}$ - the larger implication from this study is that other materials with such properties may yet to be revealed.

The authors acknowledge M. Grayson and B. Cui for very helpful comments. This material is based upon work supported by U.S. Department of Energy (DOE)/Basic Energy Sciences (BES) Grant No. DE-FG02-12ER46888 (University of Miami), the National Science Foundation under grant DMR-0907036 (Montana State University), and in Lorena by the CNPq (308162/2013-7) and FAPESP (2009/54001-2).
* Corresponding Author: cohn@physics.miami.edu

[1] H. J. Goldsmid, J. Electron. Mater. 40, 1254 (2011).

[2] C. Reitmaier, F. Walther, and H. Lengfellner, Appl. Phys. A 99, 717 (2010).

[3] C. Zhou, S. Birner, Y. Tang, K. Heinselman, and M. Grayson, Phys. Rev. Lett. 110, 227701 (2013).

[4] K. P. Ong, D. J. Singh, and P. Wu, Phys. Rev. Lett. 104, 176601 (2010); K. P. Ong, J. Zhang, John S. Tse, and Ping Wu, Phys. Rev. B 81, 115120 (2010).

[5] C. Uher, R. Hockey, and E. Ben-Jacob, Phys. Rev. B 35, 4483 (1987).

[6] N. E. Hussey et al., Phys. Rev. B 57, 5505 (1998).

[7] I. Terasaki, Y. Sasago, and K. Uchinokura, Phys. Rev. B 5, R12685 (1997).

[8] J. G. Analytis et al., Phys. Rev. Lett. 96, 177002 (2006).

[9] N. E. Hussey, M. N. McBrien, L. Balicas, and J. S. Brooks, Phys. Rev. Lett. 89, 086601 (2002).

[10] Z. Q. Mao et al., Phys. Rev. Lett. 90, 186601 (2003).

[11] W. Kobayashi, Phys. Rev. B 79, 155116 (2009).

[12] D. B. Gutman and D. L. Maslov, Phys. Rev. B 77, 035115 (2008); Phys. Rev. Lett. 99, 196602 (2007).

[13] M. Stordeur and W. Heiliger, Phys. Stat. Sol. (b) 78, K103 (1976).

[14] C. Uher, Phys. Rev. B 25, 4167 (1982).

[15] E. S. Choi et al., Synth. Met. 120, 1069 (2001).

[16] J. W. Cochrane, A. Hartmann, and G. J. Russell, Physica C 265, 135 (1996).

[17] T. Fujii, I. Terasaki and A. Matsuda, Physica C 408-410, 674 (2004).

[18] G. D. Tang et al., Appl. Phys. Lett. 98, 202109 (2011).

[19] M. Greenblatt, Chem. Rev. 88, 31 (1988).

[20] W. H. McCarroll and M. J. Greenblatt, Sol. St. Chem. 54, 282 (1984).

[21] M. H. Whangbo and E. Canadell, J. Am. Chem. Soc. 110, 358 (1988).

[22] See L. Dudy et al., J. Phys. Condens. Matter 25, 014007 (2013) and references therein.

[23] Z. S. Popović and S. Satpathy, Phys. Rev. B 74, 045117 (2006).

[24] J. Choi et al., Phys. Rev. B 69, 085120 (2004).

[25] C. A. M. dos Santos et al., Phys. Rev. Lett. 98, 266405 (2007).

[26] J. Merino and R. H. McKenzie, Phys. Rev. B 85235128 (2012).

[27] T. Jarlborg, P. Chudzinski, and T. Giamarchi, Phys. Rev. B 85, 235108 (2012); P. Chudzinski, T. Jarlborg, and T. Giamarchi, ibid. 86, 075147 (2012).

[28] M. Nuss and M. Aichhorn, Phys. Rev. B 89, 045125 (2014).

[29] See J. L. Cohn, B. D. White, C. A. M. dos Santos, and J. J. Neumeier, Phys. Rev. B 86, 195143 (2012) and references therein.

[30] J. L. Cohn, B. D. White, C. A. M. dos Santos, and J. J. Neumeier, Phys. Rev. Lett. 108, 056604 (2012).

[31] J.-F. Mercure, et al., Phys. Rev. Lett. 108, 187003 (2012).

[32] For additional data and analysis, see Supplementary Material at http://link.aps.org/supplemental/ xx.xxxx/PhysRevLett.10x.xxxxxx, which includes Ref.'s [38, 39].

[33] N. Kumar and A. M. Jyannavar, Phys. Rev. B 45, 5001 
(1992).

[34] B. Fisher and M. Fibich, Phys. Rev. B 37, 2820 (1988).

[35] T. W. Silk, I. Terasaki, T. Fujii, and A. J. Schofield, Phys. Rev. B 79, 134527 (2009).

[36] Other materials with large $Z_{x y} T$ include $\mathrm{CsBi}_{4} \mathrm{Te}_{6}$ and ReSi ${ }_{1.75}$. D.-Y. Chung, et al., Mat. Res. Soc. Symp. Proc., 793, S6.1.1 (2004); J-J Gu, et al. Mat. Res. Soc. Symp. Proc. 753, BB6.10.1 (2003); Yang Tang, Boya Cui, Chuanle Zhou, and M. Grayson, unpublished.

[37] Since $\rho_{x x}$ was not measured for the Peltier crystal, we computed it using the comprehensive results for $\rho_{b}$ from Wakeham et al. [Nature Commun. 2, 396 (2011)], lin- early extrapolated to $450 \mathrm{~K}$, and the $\rho_{c}$ data for crystal B [Fig. 1 (a)]. Using the appropriate tensor expressions (Ref. 2), this gave $\rho_{x x} \simeq 4.62 \times 10^{-5} \Omega \mathrm{m}$. The linearity in $\Delta T(I)$ (Fig. 3) implies that the actual $\rho_{x x}$ for our specimen cannot be much larger than this value (we estimate not by more than 30\%); a deviation from linearity (suppressed $\Delta T$ ) that is quadratic in $j$ would be the signature of Joule heating from the second term in the heat flux equation [2].

[38] H. Fritzsche, Sol. St. Commun. 9, 1813 (1971).

[39] V. A. Johnson and K. Lark-Horovitz, Phys. Rev. 92, 226 (1953). 


\section{Supplementary Material: Extreme thermopower anisotropy and interchain transport in the quasi-one-dimensional metal $\mathrm{Li}_{0.9} \mathrm{Mo}_{6} \mathrm{O}_{17}$}

J. L. Cohn, S. Moshfeghyeganeh, C. A. M. dos Santos, J. J. Neumeier

Additional Data for $S_{c}$ and $\rho_{c}$

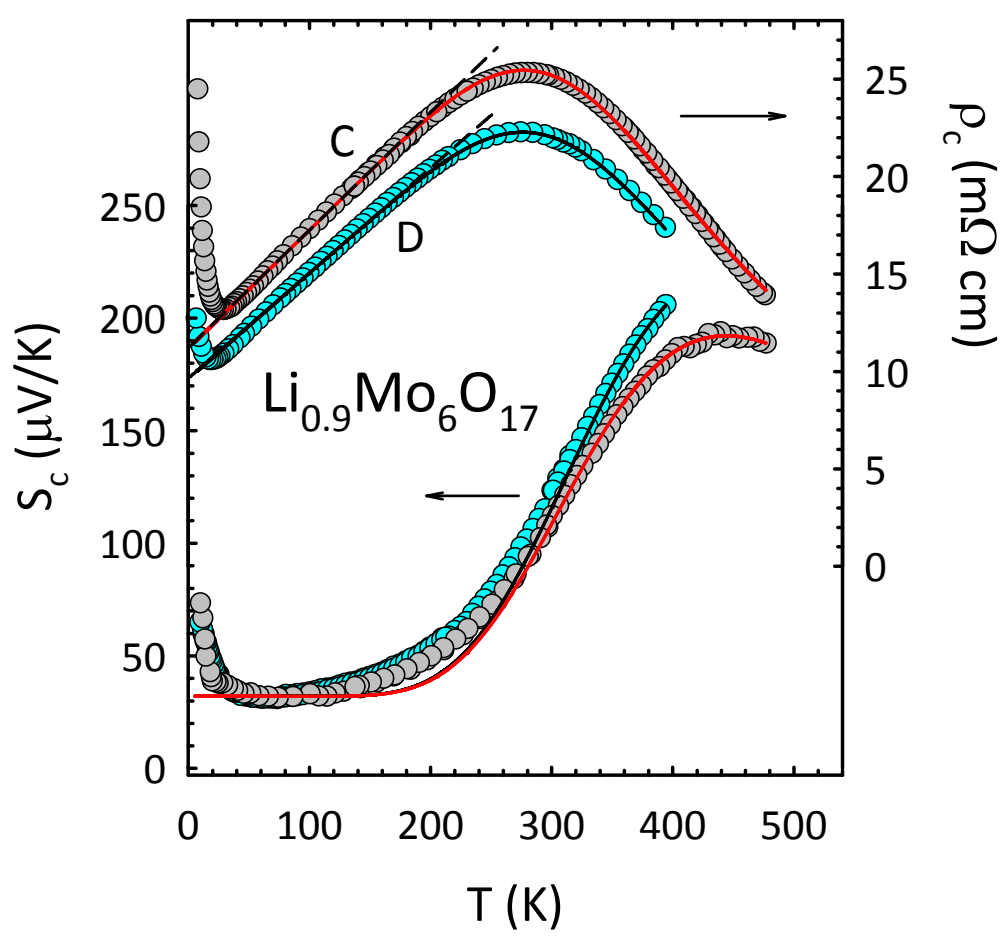

FIG. S1. LiPB inter-chain (c-axis) thermopowers (left ordinate) and resistivities (right ordinate) for crystals C and D. Solid curves through the data are fits to the parallel conduction model discussed in the text; parameters for the semiconducting component are listed in Table I. Dashed lines are linear-least-squares fits to the low- $T \rho_{c}(T)$.

Differing excitation energies for valence and conduction bands yields $E_{S}>E_{\sigma}$

Here we demonstrate that a single semiconductor component with $E_{S}>E_{\sigma}$, as emerged from the two-component model (metal + semiconductor) discussed in the text, can be reproduced by a two-component semiconductor with differing hole and electron excitation energies, $\Delta_{V}=E_{F}-E_{V}$ and $\Delta_{C}=E_{F}-E_{C}$, respectively, and $\Delta_{V}<\Delta_{C}$.

Distinguishing electron $(e)$ and hole $(h)$ contributions, we have:

$$
\begin{aligned}
\sigma & =\sigma_{e}+\sigma_{h}=C_{C} \exp \left(\Delta_{C} / k_{B} T\right)+C_{V} \exp \left(\Delta_{V} / k_{B} T\right) \\
S_{e} & =-\frac{k_{B}}{e}\left(\frac{\Delta_{C}}{k_{B} T}+A_{e}\right) ; S_{h}=\frac{k_{B}}{e}\left(\frac{\Delta_{V}}{k_{B} T}+A_{h}\right) \\
S & =\left(\sigma_{e} / \sigma\right) S_{e}+\left(\sigma_{h} / \sigma\right) S_{h} .
\end{aligned}
$$

Figure S2 shows $\sigma(T)$ and $S(T)$ computed for the following parameters appropriate to crystal B: $\Delta_{C}=0.219 \mathrm{eV}, \Delta_{V}=$ $0.135 \mathrm{eV}, C_{C}=1900 \Omega^{-1} \mathrm{~cm}^{-1}, C_{V}=985 \Omega^{-1} \mathrm{~cm}^{-1}, A_{e}=2.80$, and $A_{h}=2.69$. The solid lines demonstrate that this set of parameters produces effective single-component parameters identical to those of the single semiconductor component listed in Table I for specimen B and plotted in Fig. 1. The constants $A_{e}$ and $A_{h}$ represent weighted 
TABLE S2. Fitting parameters for the semiconducting components of the three-component model for three specimens.

\begin{tabular}{ccccccc}
\hline \hline Specimen & $C_{C}\left(\Omega^{-1} \mathrm{~cm}^{-1}\right)$ & $\Delta_{C}(\mathrm{eV})$ & $C_{V}\left(\Omega^{-1} \mathrm{~cm}^{-1}\right)$ & $\Delta_{V}(\mathrm{eV})$ & $A_{e}$ & $A_{h}$ \\
$\mathrm{~B}$ & $2.30 \times 10^{3}$ & 0.231 & $1.40 \times 10^{3}$ & 0.146 & 2.70 & 2.20 \\
$\mathrm{C}$ & $2.00 \times 10^{3}$ & 0.237 & $1.10 \times 10^{3}$ & 0.137 & 2.62 & 1.74 \\
$\mathrm{D}$ & $2.00 \times 10^{3}$ & 0.224 & $1.32 \times 10^{3}$ & 0.138 & 2.60 & 2.70 \\
\hline \hline
\end{tabular}

averages over the charge carriers in the conduction and valence bands [38]. For example, $A=3$ corresponds to a density of states and mobility that increase linearly with energy, and $A=1$ for constant density of states and mobility. Temperature dependent band energies, varying linearly in $T$ to lowest order, can contribute to these constant terms and even alter their sign [39].

\section{Three-component fitting}

Incorporating two semiconductor components along with the low- $T$ term described in the text constitutes a threecomponent model with appropriate weighting by the respective partial conductivities. This procedure produces fits that are indistinguishable from those of Fig. 1; Table S1 lists fitting parameters describing the semiconducting components for three crystals. Crystal A was excluded because its data do not extend to high enough temperature to sufficiently constrain the semiconducting parameters. Note that this analysis yields values for $\Delta_{C}$ and $\Delta_{V}$ that are only $5-10 \%$ smaller than $E_{S}$ and $E_{\sigma}$, respectively, of the simpler (two-component) model (Table I). Figure S3 shows the $T$-dependent weights (fractional conductivities) for each of the three components using the fitting parameters for crystal B.

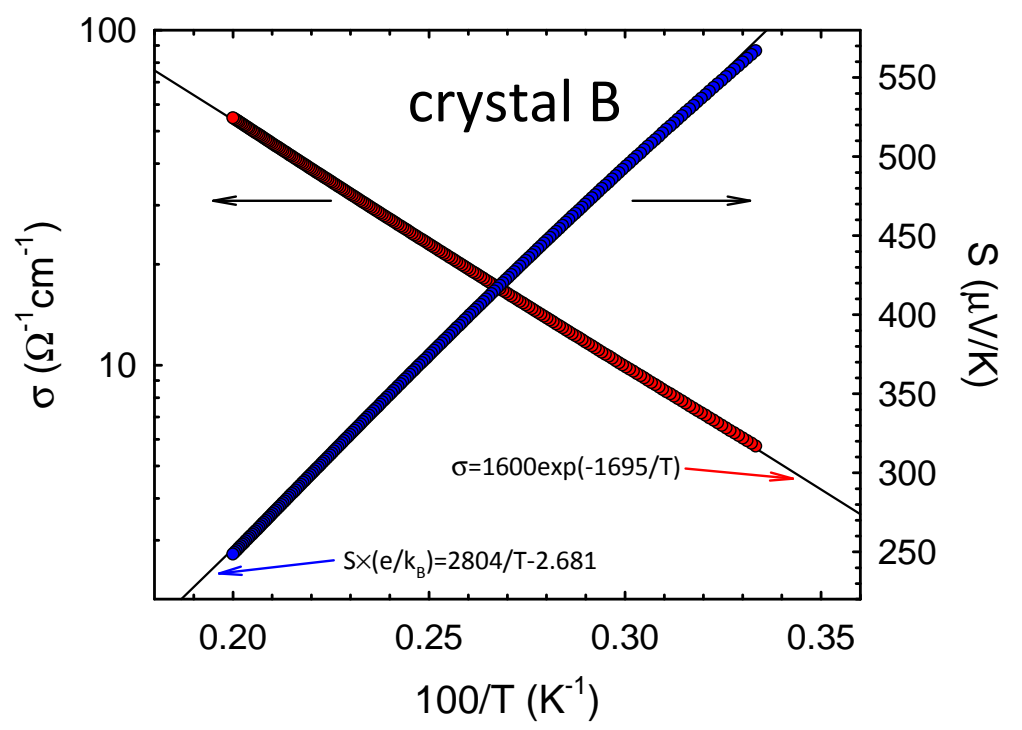

FIG. S2. Conductivity and thermopower computed from the two-component semiconductor model having effective activation energies (solid lines) matching those of the single-component semiconductor contribution to the fitting to specimen B discussed in the text (Table I). 


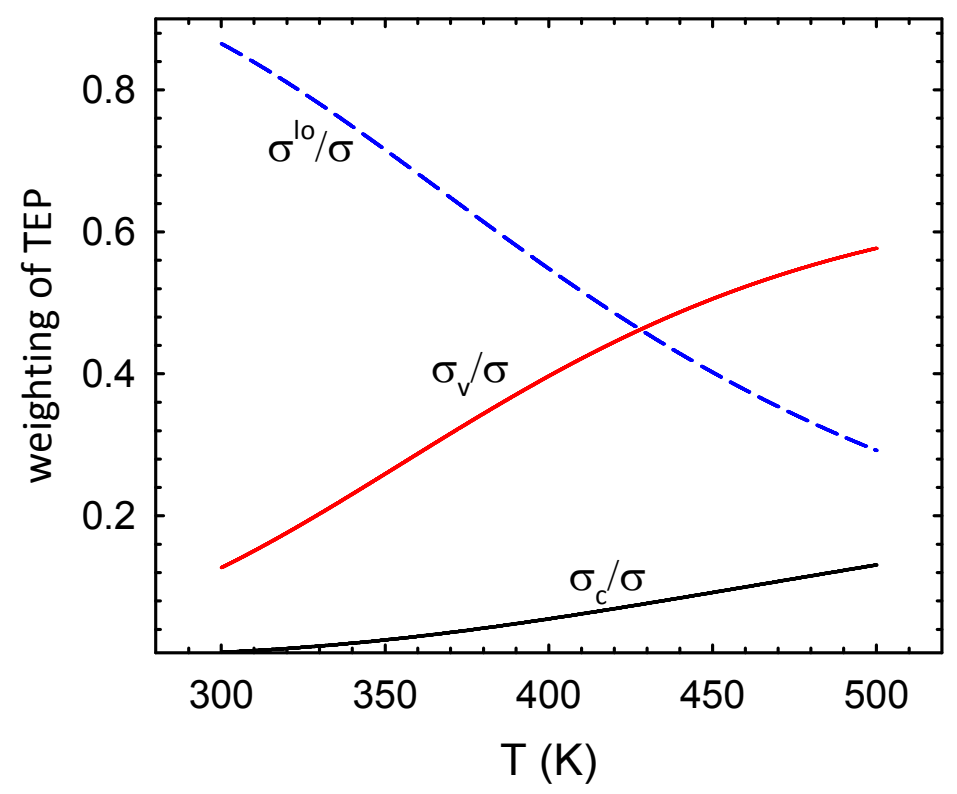

FIG. S3. T-dependent weights (conductivity ratios) for each of the three components from three-component fitting to crystal B (parameters from Table S1).

[38] H. Fritzsche, Sol. St. Commun. 9, 1813 (1971).

[39] V. A. Johnson and K. Lark-Horovitz, Phys. Rev. 92, 226 (1953). 